\title{
Correction to: Biallelic Variants in the Nuclear Pore Complex Protein NUP93 Are Associated with Non-progressive Congenital Ataxia
}

\section{Ginevra Zanni ${ }^{1}$ (D) P. De Magistris ${ }^{2} \cdot$ M. Nardella ${ }^{1} \cdot$ E. Bellacchio $^{3} \cdot$ S. Barresi ${ }^{3} \cdot$ A. Sferra $^{1} \cdot$ A. Ciolfi $^{3} \cdot$ M. Motta $^{3}$.} H. Lue $^{2} \cdot$ D. Moreno-Andres ${ }^{2} \cdot$ M. Tartaglia ${ }^{3} \cdot$ E. Bertini ${ }^{1} \cdot$ Wolfram Antonin $^{2}$

Published online: 5 March 2019

(C) Springer Science+Business Media, LLC, part of Springer Nature 2019

\section{Correction to: Cerebellum \\ https://doi.org/10.1007/s12311-019-1010-5}

The original version of this article unfortunately contained mistake in Fig. 3 image.

The online version of the original article can be found at https://doi.org/ 10.1007/s12311-019-1010-5

\section{Ginevra Zanni ginevra.zanni@opbg.net \\ $\triangle$ Wolfram Antonin \\ wantonin@ukaachen.de}

1 Department of Neurosciences, Unit of Neuromuscular and Neurodegenerative Disorders, Bambino Gesù Children's Hospital, IRCCS, 00146 Rome, Italy

2 Institute of Biochemistry and Molecular Cell Biology, Medical School, RWTH Aachen University, 52074 Aachen, Germany

3 Genetics and Rare Diseases Research Division, Bambino Gesù Children's Hospital, IRCSS, Rome, Italy 
Fig. 3 The NUP93 p.R537W variant interferes with integration into nuclear pore complexes. a HeLa cells were transfected with different EGFP-NUP93 fusion constructs, fixed $24 \mathrm{~h}$ post transfection and stained with the NPC marker mAB414 (red) and DAPI. The EGFP-NUP93 signal is shown in green. Scale bar $10 \mu \mathrm{m}$. b HEK297 cells were mock transfected or with indicated EGFP-NUP93 fusion constructs. Immunoprecipitates and 5\% of the inputs from cell lysates generated $24 \mathrm{~h}$ post-transfection were analyzed by western blotting for the NUP93 interactors NUP188 and NUP205. NUP98 served as a negative control for a noninteracting nucleoporin a

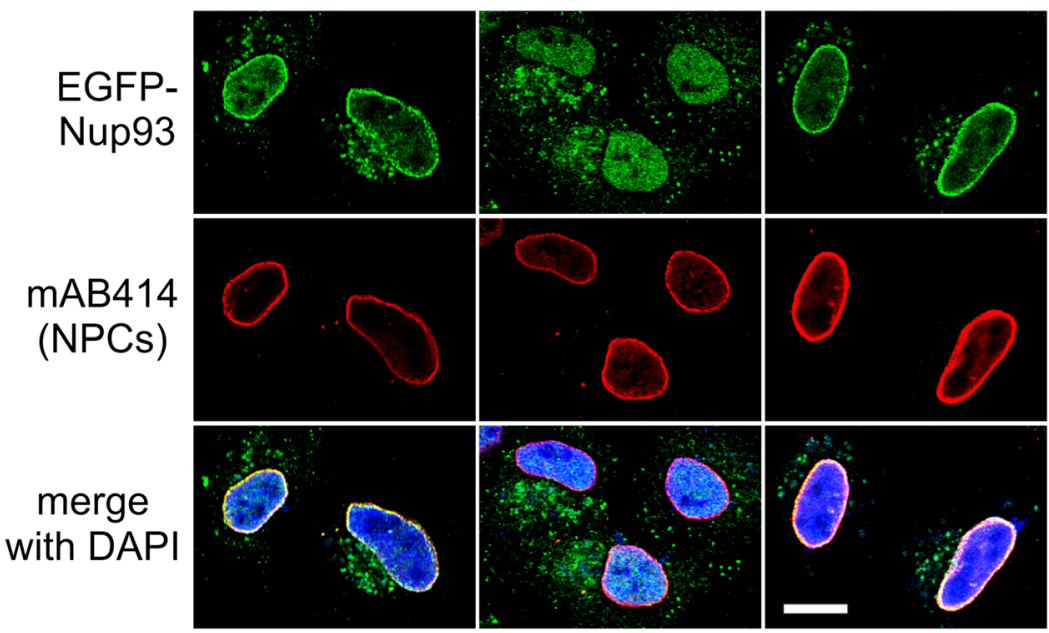

b input EGFP-pulldown

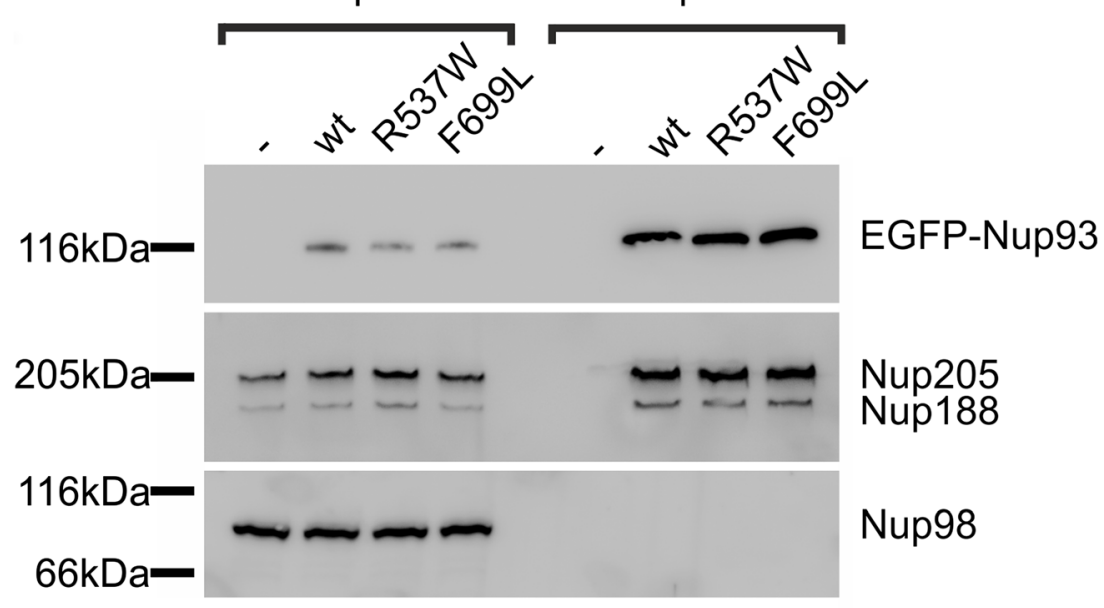

The western blot presented in Figure b shows white lines in the published version not seen in the original picture. With this, the correct Fig. 3 is hereby published.

Also the authors would like to replace the ESM3 of the article.

The original article has been corrected.

Publisher's Note Springer Nature remains neutral with regard to jurisdictional claims in published maps and institutional affiliations. 